\title{
Theranostic applications of nanoparticles in neurodegenerative disorders
}

This article was published in the following Dove Press journal:

International Journal of Nanomedicine

\author{
Sahana Ramanathan' \\ Govindaraju Archunan ${ }^{2}$ \\ Muthusamy Sivakumar ${ }^{3}$ \\ Subramanian Tamil Selvan ${ }^{4}$ \\ A Lenin Fred ${ }^{5}$ \\ Sundramurthy Kumar' \\ Balázs Gulyás' \\ Parasuraman Padmanabhan' \\ 'Lee Kong Chian School of Medicine, \\ Nanyang Technological University, \\ Singapore; ${ }^{2}$ Department of Animal \\ Science, Centre for Pheromone \\ Technology (CPT), Bharathidasan \\ University, Tiruchirappalli, India; \\ ${ }^{3}$ Nanoscience and Technology, \\ Anna University - BIT Campus, \\ Tiruchirappalli, India; ${ }^{4}$ Institute of \\ Materials Research and Engineering, \\ Singapore; ${ }^{5}$ Mar Ephraem College \\ of Engineering and Technology, \\ Kanyakumari, India
}

Correspondence: Parasuraman Padmanabhan Lee Kong Chian School of Medicine, Nanyang Technological University, 59 Nanyang Drive, 63692 I Singapore Tel +656904 I I 86

Email ppadmanabhan@ntu.edu.sg

Balázs Gulyás

Lee Kong Chian School of Medicine, Nanyang Technological University, 59 Nanyang Drive, 63692I Singapore Tel +656904 II84

Email balazs.gulyas@ntu.edu.sg
Abstract: The preeminent treatments for neurodegenerative disease are often unavailable due to the poor accessibility of therapeutic drugs. Moreover, the blood-brain barrier (BBB) effectively blocks the transfer of cells, particles and large molecules, ie, drugs, across the brain. The most important challenge in the treatment of neurodegenerative diseases is the development of targeted drug delivery system. Theranostic strategies are known to combine therapeutic and diagnostic capabilities together. The aim of this review was to record the response to treatment and thereby improve drug safety. Nanotechnology offers a platform for designing and developing theranostic agents that can be used as an efficient nano-carrier system. This is achieved by the manipulation of some of the properties of nanoparticles (NPs), thereby enabling the attachment of suitable drugs onto their surface. The results provide revolutionary treatments by stimulation and thus interaction with targeted sites to promote physiological response with minimum side effects. This review is a brief discussion of the administration of drugs across the brain and the advantages of using NPs as an effective theranostic platform in the treatment of Alzheimer's, Parkinson's, epilepsy and Huntington's disease.

Keywords: theranostics, neurological disease, nanoparticles, imaging

\section{Introduction}

Nanotechnology involves the manipulation of particles at the atomic and molecular level with at least one dimension within $100 \mathrm{~nm}$. The properties such as physical, chemical, biological, size distribution, morphology and characteristics are unique, and when compared with bulky materials, their effectiveness in localizing the cells is significant. In the past, noble metal nanoparticles (NPs), magnetic NPs and quantum dots (QDs) were employed for various scientific and therapeutic purposes including drug delivery, for assays of metal ions, proteins and nucleic acid biomarkers. ${ }^{1,2}$ Recent developments in nanotechnology have created an opportunity to bring diagnosis and therapeutics to a nearby platform. NP-based therapies have been rigorously studied to discover the starting point for the development of theranostics. Some of the NPs have been used as imaging agents and have the potential for future theranostic agents and can act in two ways. Mostly, they are employed in disease management to overcome the drawbacks of conventional NP system such as patient compliance and the safety. The term "theranostics" was used to denote more specific and individualized therapies for various diseases. The synthesis of nano-sized carriers composed of lipids, polymers, carbon or metals which are being developed has overcome the limitations faced during the traditional drug delivery methods. ${ }^{3-7}$ The term "neurodegenerative disorders" (NDs) represents conditions, which are intermittent and familial and are further characterized by the gradual loss of acuity in thought processes, memory and skilled movements. 
NDs share some similarities in subcellular and molecular level such as synaptic abnormalities, neuronal loss and occurrence of cerebral deposits, which are caused by the aggregation of misfolded proteins. Yet, there are important differences at the subcellular level from other diseases affecting the brain such as cancer or stroke. ${ }^{8,9}$ NDs are prevalent and lead to short- and long-term impairments. Moreover, such impairments are an emotional, social and financial burden on the sufferer and their families. This review focuses on methods for the successful delivery of drugs across the blood-brain barrier (BBB) for the treatment of central nervous system (CNS)-related disorders. An overview of NPs as an effective theranostic platform in the treatment of NDs such as Alzheimer's disease (AD), Parkinson's disease (PD), epilepsy and Huntington's disease (HD) is presented.

Theranostic nanomedicine is an emerging field as a promising approach in therapeutic paradigm. The term "theranostics" was coined to define ongoing efforts in clinics to develop more specific individualized therapies for various diseases and to combine diagnostic and therapeutic capabilities into a single agent. The emergence of nanotechnology has offered a platform to combine diagnosis and therapy closer. Magnetic resonance imaging (MRI), positron-emission tomography and single-photon emission computed tomography are available to track the theranostics and clinical nuclear imaging systems upon successful delivery.

Theranostics were extensively used in imaging and therapy and also for the development of targeted drug delivery systems toward cancer, diabetes, CNS disorders, immune disorders and genetic disorders.

\section{BBB}

Various drugs with different mechanisms and targets have been developed for the treatment of CNS disorders; yet, the rate of success has been found to be negligible. The lack of efficacy may be attributed to the BBB, the nonselective distribution of the drugs in the brain, the opsonization of plasma proteins in the proper circulation and peripheral side effects for the release of drugs. NPs have gained acclaim due to the ease of their penetration into the CNS; for example, various polymeric, lipid and inorganic NPs aid in drug delivery and drug release for several CNS conditions. ${ }^{10,11}$ BBB separates the circulating blood from the brain and extracellular fluid in the CNS, to facilitate selective passage. To develop an efficient drug delivery method, it is important to understand the physiological characters of these barriers. The walls of capillaries present in the BBB separate the brain from circulating blood. ${ }^{12,13}$

Figure 1 shows the various mechanisms of transport across the BBB. Due to the lack of fenestrated and intracellular cleft, BBB does not permit the entry of solutes from the periphery to the brain. The endothelial cells in the brain-blood vessel have compact circumferential junctions in between them, and so the paracellular pathways among the cells are abolished. Thus, the transportation of polar solutes through the cerebral endothelium is hindered by

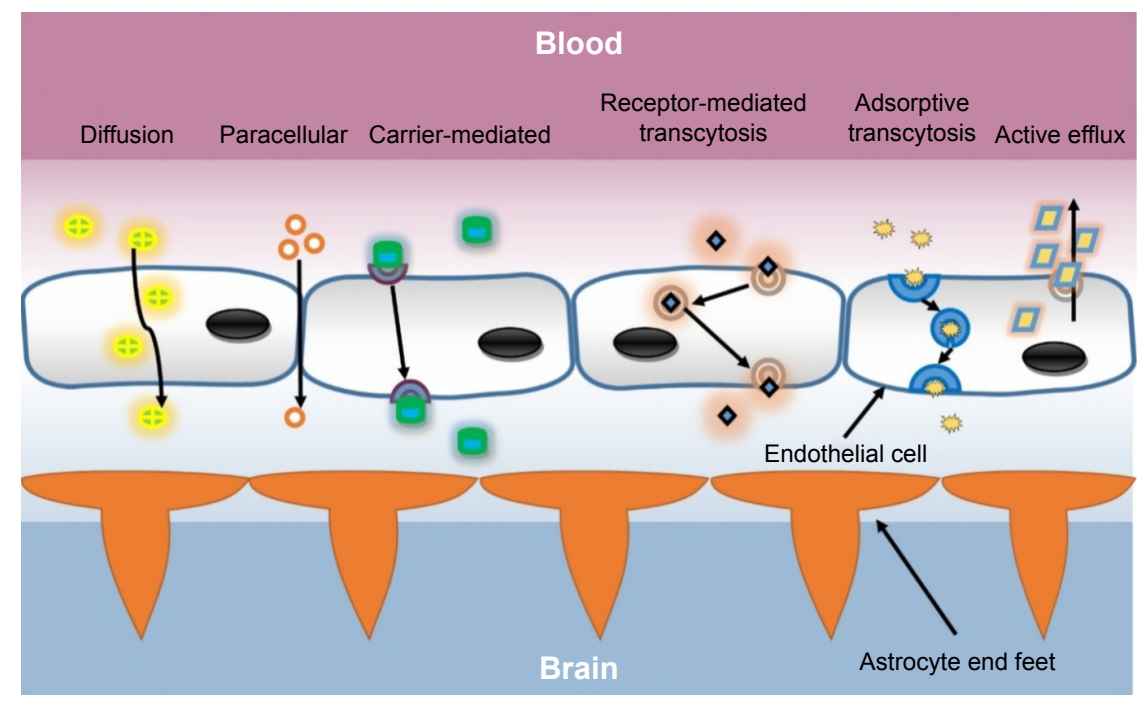

Figure I Various mechanisms of transport across BBB.

Abbreviation: BBB, blood-brain barrier. 
the occurrence of tight junction and the absence of aqueous pathway among the cells.

The various mechanisms to transport the molecules across BBB involve the following:

1. Diffusion: some small lipophilic and low molecular weight molecules ( $<500 \mathrm{Da})$ can diffuse passively across the BBB. ${ }^{14}$

2. Paracellular diffusional pathways: $B B B$ disruption due to local inflammation by CNS and circulatory factors leads to weakening of the tight junction, which allows the entry of polar solutes between the endothelial cells from blood vessels to brain extracellular fluid. ${ }^{14}$

3. Carrier-mediated transport: small molecules such as ions, amino acids and glucose can be transferred from blood vessels to brain extracellular space through endothelial cells by the carrier protein in the presence of plasma membrane. ${ }^{11}$

4. Receptor-mediated transcytosis: larger and/or hydrophilic molecules such as hormones and proteins can be transported by the specific receptors, expressed on the luminal side of the endothelial cells, in the brain parenchyma. $^{2}$

5. Adsorptive transcytosis: this mechanism involves the endocytosis of charged substance vesicles, similar to receptor-mediated transcytosis, but it is not a specific mechanism. ${ }^{2,14}$

Lipophilicity and molecular weight are the two important factors influencing the passive transport of particles through the brain endothelial cells. Even though several varieties of drugs possess the property of lipophilicity, drugs are spontaneously reversed to the blood stream. This is mainly due to the presence of effective efflux pumps such as multispecific organic anion transporter (MOAT), P-glycoprotein (Pgp) and multidrug resistance proteins (MRPs). ${ }^{15,16}$ The field of nanotechnology is actively involved in developing a promising strategy for successful drug delivery system across the BBB.

\section{NPs}

NPs are generally regarded as particles with size smaller than $100 \mathrm{~nm}$. They exist in either amorphous or crystalline form and have the ideal properties such as active surface (for catalytic properties), identical strength (for resistance against crush) and discrete energy levels (for tailoring the electronic properties). The smaller the size of NPs the more suitable they are for the drug delivery and target. NPs have comparatively high cellular uptake as compared to microparticles due to their small size and mobility, this allows NPs to target a wide range of cellular and intracellular targets. ${ }^{17}$ However, NPs sized less than $6 \mathrm{~nm}$ are prone to rapid elimination from the body via renal filtration. ${ }^{18}$ Moreover, the hydrophobicity of NPs also determines the in vivo fate of the NPs. Non-modified NPs (conventional NPs) are prone to get opsonized and considerably cleared by the mononuclear phagocyte system (MPS). To overcome this, they should be coated with the hydrophilic polymers/surfactants, or NPs should be synthesized using biodegradable copolymers with hydrophilic properties such as polyethylene oxide, polyethylene glycol (PEG), poloxamer, polysorbate 80 (Tween 80 ) and poloxamine. ${ }^{17,19}$ NPs more than $100 \mathrm{~nm}$ in size have significantly less bio-distribution and are easily captured by Kupffer cells, which leads to clearance by MPS. ${ }^{20}$

They have unique features such as fluorescence properties (due to quantum effects of QDs) and ability to absorb and transport various substances such as drugs, proteins and probes (due to their high surface to volume ratio as compared with the other macroscopic materials). The unique fluorescence properties of QDs are size dependent, as the QD size increases, the energy gap between valence and conduction bands decreases. This property allows them to absorb photon and emit specific wavelength spectra within the range from blue to red. ${ }^{21}$

Besides this, zeta potential of NPs and drug-loading capacity of the NPs also play a major role. ${ }^{2,20}$ NPs appear in different shapes such as nanocubes, nanoplates, nanorods, nanospheres, nanotetrapods, nanoprisms and nanobelts. ${ }^{22-24}$ Various types of NPs have been synthesized with different properties such as polymer NPs, liposomes, dendrimers, micelles, metal NPs and carbon nanotubes. ${ }^{25}$ Table 1 summarizes the functions of NPs in the treatment of various diseases.

An effective nano-carrier to cross the BBB should have the following characteristic features:

- The size of the particles should be lesser than $100 \mathrm{~nm}$.

- The synthesized particles should be nontoxic, biocompatible and biodegradable.

- Stability in blood (they should not be opsonized by the proteins).

- It must be targeting BBB.

- No aggregation of platelet.

- Reticuloendothelial entry should be avoided.

- Many NPs cannot diffuse freely across the BBB, thus they should have a receptor-mediated transport mechanism. The advancements made in the field of nanotechnology have been able to overcome the problems related to the cell 
Table I Functions of NPs in the treatment of various diseases

\begin{tabular}{|c|c|c|c|}
\hline NPs & Disease & Role & Reference \\
\hline Lipid-polymer hybrid NPs & $\begin{array}{l}\text { Treatment of pancreatic } \\
\text { cancer }\end{array}$ & $\begin{array}{l}\text { Delivery of HIFI } \alpha \text { siRNA and gemcitabine. Through the enhanced } \\
\text { vasculature effect in tumor cells, the drug release is improved }\end{array}$ & 32 \\
\hline Porous silicon NPs & Treatment of cancer & $\begin{array}{l}\text { Dextran-coated silicon NPs were employed as sonosensitizers for } \\
\text { suppressing the cancer cell proliferation }\end{array}$ & 33 \\
\hline Bovine serum albumin NPs & $\begin{array}{l}\text { Prevention of acute rejection } \\
\text { of organ transplants }\end{array}$ & $\begin{array}{l}\text { NPs used as drug delivery vehicle for controlling the distribution of } \\
\text { NPs and reducing the functional nephrotoxicity }\end{array}$ & 34 \\
\hline Gold NPs & $\begin{array}{l}\text { Treatment of cardiovascular } \\
\text { disorders }\end{array}$ & $\begin{array}{l}\text { Gold NPs were functionalized with malondialdehyde-modified } \\
\text { low-density lipoprotein antibodies. For capturing and recognizing } \\
\text { MDA-LDL presents in biological matrices }\end{array}$ & 35 \\
\hline PLGA-PEG NPs & Treatment of bone & $\begin{array}{l}\text { NP-targeting bone based on dendritic trimer in aspartic acid } \\
\text { oligopeptide }\left(\mathrm{Asp}_{3}\right) \text {, conjugated to PLGA-PEG copolymers. It was } \\
\text { found to have the ability to accumulate in bone }\end{array}$ & 36 \\
\hline Selenium NPs & $\begin{array}{l}\text { Treatment of diabetic } \\
\text { nephropathy }\end{array}$ & $\begin{array}{l}\text { Quenches oxidative stress and also activates longevity protein SIRTI } \\
\text { and cytoprotective protein HSP70 }\end{array}$ & 37 \\
\hline $\begin{array}{l}\text { Poly(y-benzyl-L-glutamate)-PEG- } \\
\text { alendronate multivalent NPs }\end{array}$ & Skeletal problems & NPs increase the strength of interaction with hydroxyapatite & 38 \\
\hline $\begin{array}{l}\text { Super paramagnetic iron oxide } \\
\text { NPs }\end{array}$ & Imaging myocardial infarction & NPs employed as macrophage-targeting molecular imaging agents & 39 \\
\hline Silica NPs & $\begin{array}{l}\text { Treatment of breast and } \\
\text { prostate cancer }\end{array}$ & $\begin{array}{l}\text { Snake venom as antitumor drug loaded in the NPs. Elevation in the } \\
\text { free radical level and apoptosis was found in the tumor cells }\end{array}$ & 40 \\
\hline SLNs & Cardio-cerebrovascular & $\begin{array}{l}\text { NPs loaded with daidzein. SLN increases the circulating time and } \\
\text { reduces the myocardial oxygen consumption and coronary resistance }\end{array}$ & 41 \\
\hline
\end{tabular}

Abbreviations: NPs, nanoparticles; PEG, polyethylene glycol; PLGA, poly(lactic-co-glycolic acid); SLN, solid lipid NP.

therapy approaches. In addition, recent study in NPs has made the materials compatible, and development procedures have driven this field closer to the clinical applications. Other small molecules that are being used in the conventional treatment for various neurological diseases have limitations such as lack of water solubility, nonspecific targeting and bio-distribution as well as less therapeutic indices. Moreover, conventional treatment can also result in the development of drug resistance, which further affects the efficacy of newly targeted therapeutic agent that limits their practice in clinical applications. ${ }^{26}$

Figure 2 shows various advantages and disadvantages of NPs in theranostic applications. The administration of these NP drugs into the brain can be achieved only if they can pass through the BBB. Various chemical and biological methods have been employed for successful drug delivery. Specific endogenous BBB transporters are available for some of the molecules essential for the functioning of brain such as amino acids, peptides, glucose and proteins, and these transporters can transport molecules smaller than $600 \mathrm{Da}$. Carrier-mediated transcytosis is employed for the delivery of nutrients, mainly amino acids, purine bases and glucose. They are substrate-selective inhibitors and can transfer the drugs which mimic endogenous carrier substrates. The transportation of big molecules such as lipoprotein and antibodies is through the receptor-/adsorptive-mediated endocytosis. This process starts upon the binding of receptor-specific ligand, followed by the binding and internalization of the substance. Some of the approaches considered for drug delivery routes are via oral route, inhalation or intra-tracheal installation and intravenous
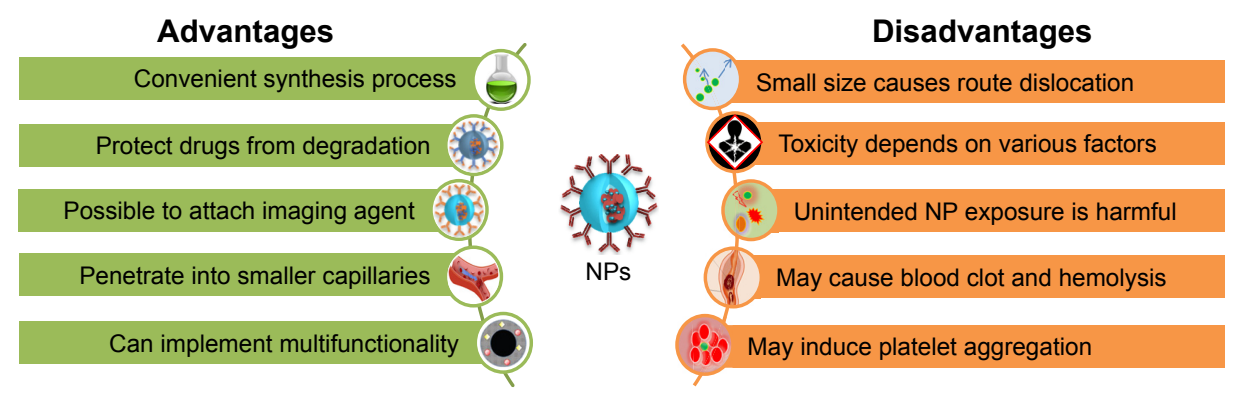

Figure 2 Various advantages and disadvantages of NPs in theranostic applications. Abbreviation: NP, nanoparticle. 
(IV) route. The entry of NPs into the brain involves two different pathways. In the first one, the NPs are translocated to the CNS by the uptake of NPs either through sensory nerve endings that present at the airway epithelia or by the nerve endings of the olfactory bulb. However, most of the studies have to be performed with nonhuman sources, and therefore further advancement is needed to develop human models for predicting and evaluating the fate of NPs during their transport. Addressing safety concern is also very critical to move this study field to potential clinical treatment. However, most effective fabricated NPs also have some significant undesirable accumulations of NPs in other body regions such as spleen, liver, kidney and other organs/tissues. Thus, it is very important to design and fabricate NPs that target only brain tissues selectively and can be remotely triggered for drug release, instead of delivering drug into other body parts. ${ }^{27}$

The administration of drug through nasal route is mostly preferred since the drug is protected from degradation and return to the nasal cavity. Nasal route also helps NPs by increasing the bioavailability, absorption rate and reducing the drug degradation, and NPs first-pass metabolism in the liver is prevented. ${ }^{28-31}$

\section{AD}

Today, millions of elderly people are affected by the most common ND known as AD. This neuropathological condition mainly occurs due to the accumulation of amyloid plaque caused by $A \beta$ protein aggregation and due to the presence of intracellular neurofibrillary tangles (NFTs) which are formed as a result of the aggregation of hyperphosphorylated tau protein. ${ }^{42-44}$ The disease is characterized by the loss of spatial and temporal orientation and loss of verbal fluency. In the further stages, the patients can be affected by pneumonia, decubitus ulcers and infections. ${ }^{45,46}$

Patients characterized with AD are categorized as presenile $\mathrm{AD}$ or early-onset and sporadic $\mathrm{AD}$ or late onset. In the case of presenile $\mathrm{AD}$, the symptoms are seen at an earlier age than 65 years. The main cause is the genetic mutation in the presenilin 1 (PS1) and presenilin 2 (PS2), which eliminates the $\beta \gamma$-secretase activity by increasing the production of strong self-aggregating $A \beta 42$ peptide, and the patients have positive family history. ${ }^{47-50}$ Regarding the sporadic AD, the main cause in not known. The AD is believed to be the result of gene mutations or factors, such as lifestyle, smoking, hypertension, heart disease, stroke and aging. ${ }^{51}$ Amyloid cascade hypothesis is the most widely accepted theory regarding the causes of $\mathrm{AD}$. The findings are the aggregation and accumulation of $A \beta 42$ which promotes further pathological effects such as the occurrence of NFTs, disrupting synaptic connections along with decreased release of neurotransmitters, activation of microglia and astrocytes in chronic inflammation response, which finally leads to dementia by causing neuronal loss. Later studies revealed that the non-fibrillar oligomeric $A \beta$ deposits were found to be more highly neurotoxic than the fibrillar $\mathrm{A} \beta$ deposits in the $\mathrm{AD}$ brain. ${ }^{52,53}$

Scientists are currently focused on the development of suitable nano-platforms for the imaging and effective drug delivery systems to the CNS diseases. ${ }^{14,54}$ Roney et $\mathrm{al}^{55}$ reported on the biodegradable polymeric NPs with suitable surface modifications and their ability to deliver drugs across the BBB for treating neurodegenerative diseases. Tanifum et $\mathrm{al}^{56}$ used stealth liposomal NPs for the incorporation of synthesized $A \beta$-targeted lipid conjugate toward amyloid plaque deposition in a preclinical model. They resulted in successful crossing of the BBB and binding with the deposition of $A \beta$ plaque.

Cui et $\mathrm{al}^{57}$ used NPs as an efficient drug delivery system through the systemic administration. The $\mathrm{Cu}$ (I) chelator D-penicillamine was conjugated by either disulfide bond or the ether bond to 1,2-dioleoyl-sn-glycero-3-phosphoethanolamine- $N$-[4-(p-maleimidophenyl)butyramide] (sodium salt) (MPB-PE) and 1,2-dioleoyl-sn-glycero-3phosphoethanolamine- $N$-[3-(2-pyridyldithio)-propionate] (sodium salt) (PDP-PE). The NPs have been taken up by the brain in vivo or in situ without any changes in permeability or integrity. They were also found to be stable at $\mathrm{pH}$ 6-8. In aqueous suspended medium while preserved at $4^{\circ} \mathrm{C}-8^{\circ} \mathrm{C}$, aggregation was not found with serum salts. The release of D-penicillamine from NPs was achieved by the usage of dithiothreitol as reducing agent. The efficiency in resolubilizing $A \beta$ was tested by incubating D-penicillamine conjugated to PDP-PE NPs in $\mathrm{Cu}-\mathrm{A} \beta$ (1-42) aggregates; the result showed that they were not effective. Then, followed by the addition of $0.1 \mathrm{~N} \mathrm{NaOH}$ at $45^{\circ} \mathrm{C}$ for $1 \mathrm{~h}$ to D-penicillamine conjugated to PDP-PE, NPs increased its efficiency.

A small nanovehicle (SNV) was designed by Agyare et $\mathrm{al}^{58}$ which had the ability to travel across the BBB and target the cerebrovascular amyloid which is developed in both the cases of cerebrovascular amyloid angiopathy (CAA) and AD. The SNV was of chitosan polymeric core developed by ionic gelation with tripolyphosphate (TPP) and polyamine-modified $\mathrm{F}$ ( $\mathrm{ab}^{\prime}$ ) portion of IgG (anti-amyloid antibody) biosensor. A control nanovehicle (CNV) with similar polymeric core and with bovine serum albumin coating was used. Bastus et $\mathrm{al}^{59}$ reported the combined usage of gold NPs and weak microwave fields. 
The studies concluded that the proteins and other aggregates that are the main causes of pathological disorder including $\mathrm{AD}$ can be removed.

The existence of BBB makes the cure of CNS disease challengeable. Zhang et $\mathrm{al}^{60}$ showed that the drug delivery to the brain is enhanced by administering lectin-modified PEG-poly(lactic-co-glycolic acid) (PEG-PLGA) NPs intranasally. Conjugation of Solanum tuberosum lectin (STL) to NPs with encapsulated bFGF enhances their selective binding with $N$-acetyl glucosamine on nasal epithelial membrane, for its brain delivery, which results in uniformsized, negative zeta potential NPs. Radioisotopic tracing method is used to assess the intranasal administration of brain distribution of ${ }^{125} \mathrm{I}-\mathrm{bFGF}$ in rats. Injection of ibotenic acid and $\beta$-amyloid was given intra-cerebroventricullary for neuroprotective effect, and it is evaluated using the Morris water maze test. This STL-bFGF-NP was better than other preparations for spatial learning and memory of AD rats. When compared with the value of choline acetyltransferase activity of rat hippocampal regional observation, the result remained consistent. The in vivo safety of STL-bFGF-NPs was confirmed by histopathological assay. From the results obtained, it was concluded that, in the case of peptide and protein drugs, the promising drug delivery to enter CNS was STL-NPs therapeutically.

Zhang et $\mathrm{al}^{61}$ developed targeted dual functional NPs consisting of PEG-PLGA NPs. Two targeting peptides were used at the surface of PEG-PLGA NPs. TGNC (which targets ligands at $\mathrm{BBB}$ ) was used as first-order targeting ligand, and second, QSH was employed for its excellent affinity to $A \beta 42$, which is one of the main components of amyloid plaque. A maleimide-thiol coupling reaction was performed for conjugating the TGN and QSH. The optimum molar ratio of maleimide/peptide was found to be $3: 1$. The in vivo and ex vivo images are shown in Figure 3. ${ }^{61}$

By performing thioflavin T (ThT)-binding assay, cellular uptake assay, in vivo imaging and surface plasmon resonance (SPR), the optimization of ligand density was conducted by Zhang et al. ${ }^{60}$ Through further studies of NP distribution in brain and ex vivo imaging, the dual targeting activity
A

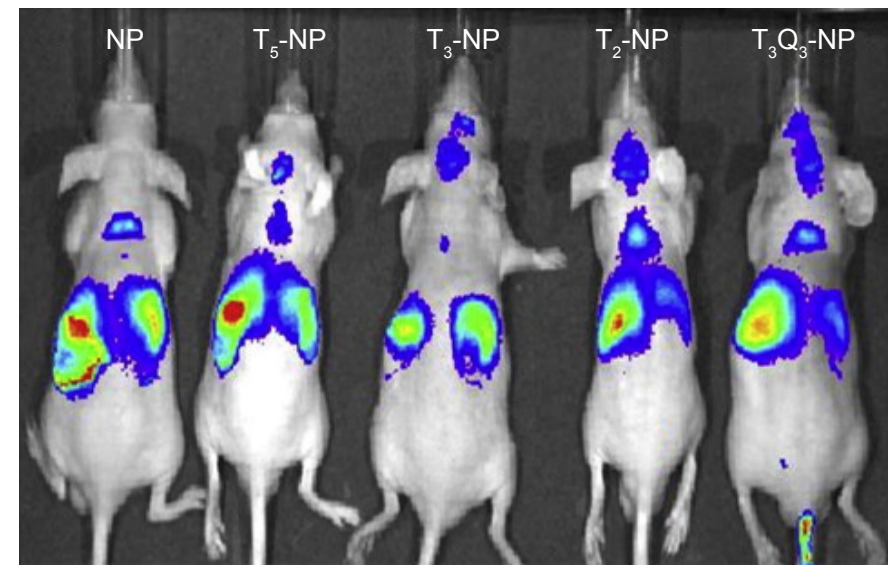

B
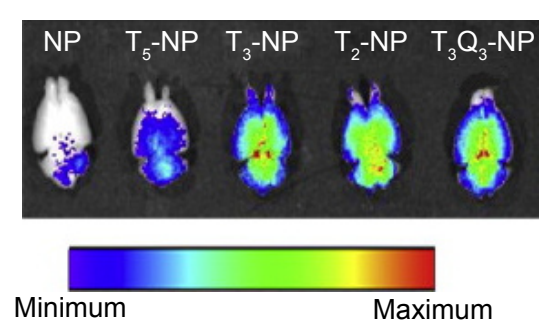

C

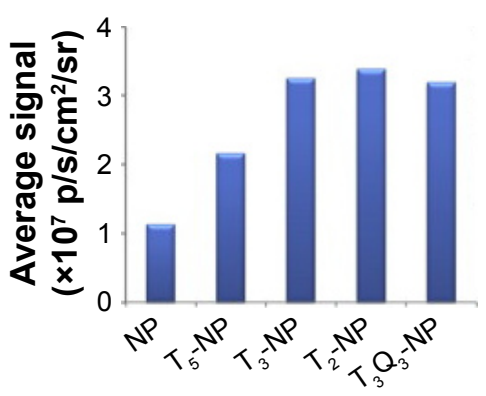

D

(i)

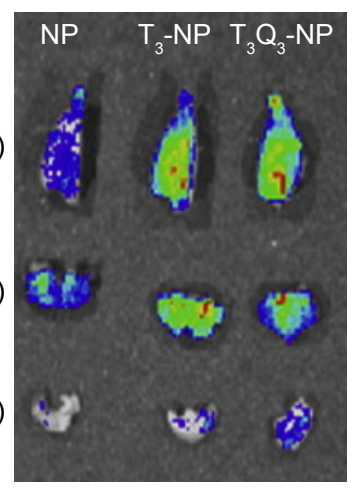

$\mathbf{E}$

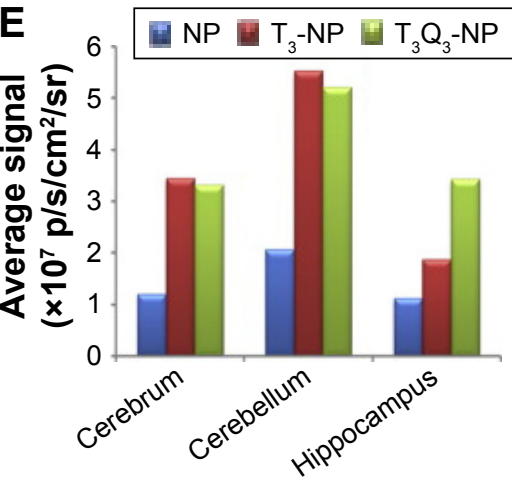

Figure 3 The in vivo $(\mathbf{A})$ and ex vivo (B, D) imaging of DiR-loaded NPs in nude mice $(\mathbf{A}, \mathbf{B})$ or $A D$ model mice $(\mathbf{D})$ and the fluorescent intensity of the brain tissues (C, E). Notes: (A) The in vivo imaging of nude mice treated with NP, $T_{5}-N P, T_{3}-N P, T_{2}-N P$ and $T_{3} Q_{3}-N P$. (B, C) The ex vivo imaging and fluorescent intensity of the brains from nude mice treated with NP, $T_{5}-N P, T_{3}-N P, T_{2}-N P$ and $T_{3} Q_{3}-N P$; (D, E) The ex vivo imaging and the fluorescent intensity of the cerebrum (i), cerebellum (ii) and hippocampus (iii) of the AD model mice treated with NP, $T_{3}-N P$ and $T_{3} Q_{3}-N P$. Adapted from Biomaterials. Zhang $C$, Wan $X$, Zheng $X$, et al. Dual-functional nanoparticles targeting amyloid plaques in the brains of Alzheimer's disease mice. 20I4;35(I):456-465. Copyright (C) 2013 Elsevier Ltd. All rights reserved, with permission from Elsevier. ${ }^{61}$ Abbreviations: AD, Alzheimer's disease; DiR, I,I'-Dioctadecyl-3,3,3',3'-tetramethylindotricarbocyanine iodide; NP, nanoparticle. 
was found. The synthesized NPs were found to enhance the successful targeted delivery toward amyloid plaque in $\mathrm{AD}$ mice model.

PEG-PLFA NPs were effective in the prevention of $A \beta 1-40$ and $A \beta 1-42$ peptides from self-aggregation and toxic oligomer formation, and besides this, due to high binding affinity toward $A \beta$ species, they showed a significant fluorescent enhancement. Figure 4 shows the theranostic effect of (E)-4-(4-(dibutylamino)styryl)-1(2-hydroxyethyl)quinolin-1-ium chloride (DBA-SLOH) with near-infrared (NIR) fluorescence images and relative fluorescence signal on transgenic (12- and 6-month-old) and wild-type mice at different time points before and after IV injection. ${ }^{62}$

In conclusion, the PEG-PLGA NPs contain two targeting peptides, namely TGNC and QSH, that have excellent affinity toward ligands present at $\mathrm{BBB}$ and $\mathrm{A} \beta 42$, which are the main components of amyloid plaque. This was confirmed by performing the ThT-binding assay, cellular uptake assay, in vivo imaging, and SPR for optimization of ligand density.
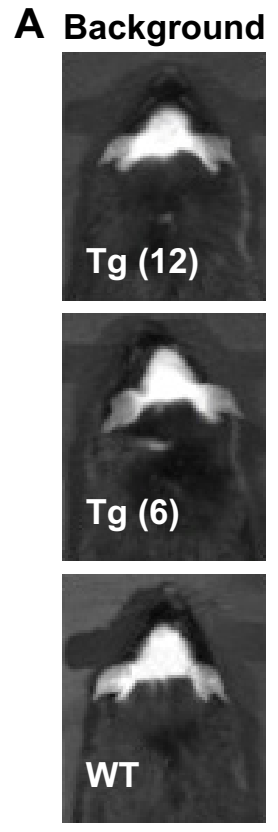
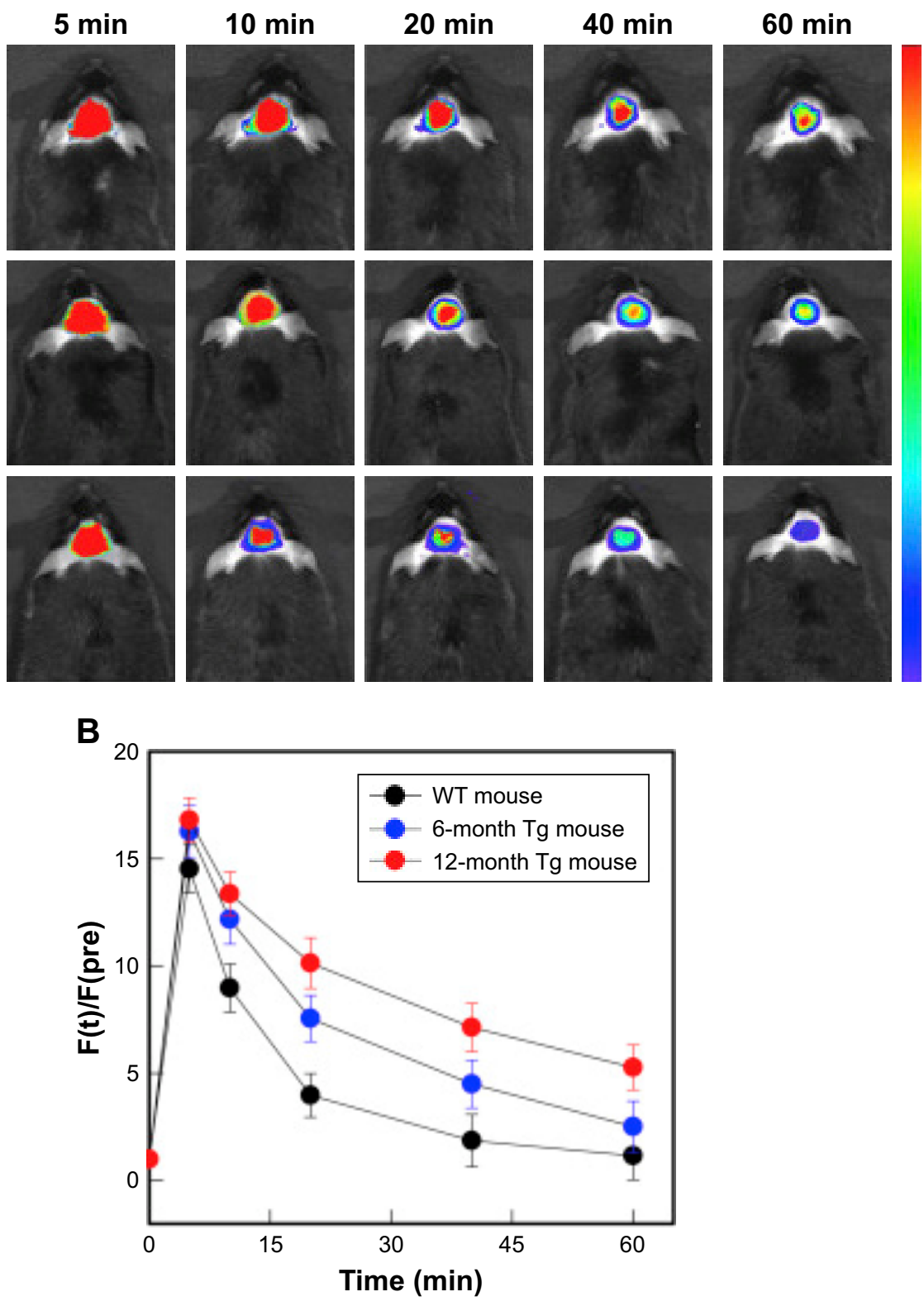

Figure 4 (A) NIR fluorescence images of Tg (12- and 6-month-old) and WT mice at different time points before and after IV injection of DBA-SLOH (5 mg.kg-1). $\lambda_{\text {ex }}=$ $535 \mathrm{~nm}, \lambda_{\text {em }}=640-680 \mathrm{~nm}$. (B) Relative fluorescence signal (F[t]/F[pre]) in the brain regions of Tg (I2-month, red), Tg (6-month, blue) and WT (black) mice after IV injection of DBA-SLOH $\left(n=3,5.0 \mathrm{mg} \cdot \mathrm{kg}^{-1}\right)$. The ( $\left.[\mathrm{t}] \mathrm{f} / \mathrm{F}[\mathrm{pre}]\right)$ of $\mathrm{Tg}$ mouse was significantly higher than that of WT mouse $(p<0.05)$. Adapted from Biomaterials. Li Y, Xu D, Ho SL, Li HW, Yang R, Wong MS. A theranostic agent for in vivo near-infrared imaging of $\beta$-amyloid species and inhibition of $\beta$-amyloid aggregation. 20I6;94:84-92. @ 20I6 Elsevier Ltd. All rights reserved, with permission from Elsevier.62

Abbreviations: DBA-SLOH, (E)-4-(4-(dibutylamino)styryl)-I-(2-hydroxyethyl)quinolin-I-ium chloride; IV, intravenous; NIR, near infrared; Tg, transgenic; WT, wild type. 


\section{Epilepsy}

Epilepsy is a CNS disorder during which the nerve cell activity in the brain is disturbed causing spontaneous and recurring epileptic seizures (paroxysmal neural discharge). Seizures are divided into generalized seizure and partial seizure. In the case of generalized seizure, both hemispheres of the brain are affected. ${ }^{63,64}$

They are further classified as follows:

- Absence seizure: consciousness is lost for a short period, and no symptoms are seen.

- Grand mal seizure: consciousness is lost, and the patient is collapsed.

- Myoclonic seizure: infrequent jerks are noticed at particular parts or in whole area of the body.

- Atonic seizure: general loss of muscle tone is noticed for $1-2 \mathrm{~s}$.

- Clonic seizure: the patients have repetitive and rhythmic jerks occurring on both the sides of the body.

- Tonic seizure: stiffening of muscles is seen.

Partial seizures are developed at specific areas of the brain. Based on etiology, epilepsy is classified as follows:

- Symptomatic: results due to genetic abnormalities.

- Idiopathic: mainly resulted due to epileptic lesions which can be a tumor or defect in metabolism causing brain injury.

- Presumed symptomatic (cryptogenic): it develops presumptive lesions.

Researchers are focusing on delivering antiepileptic drugs (AEDs) that target brain in efficient quantities to decrease the severity and the impact of seizures without any adverse effects. The current methods are designed to increase the production of AED levels in the blood through either IV injection or usage of pills. Yet again, these methods have scant penetration and entry of AED to target the brain. Encapsulation of drugs within nanomaterials is being the best alternative to the delivery of free AED. The main causes of epilepsy are the development of infection in the brain, reduced supply of oxygen to the brain after stroke or during the time of birth, head trauma, arteriovenous malformations, cerebrovascular diseases and perinatal injuries. The fluctuation of ionic concentrations such as increased $\mathrm{K}^{+}$concentrations due to the repetitive interictal and ictal activity or the decreased extracellular $\mathrm{Ca}^{++}$failure in the ion transportation are also another important cause of epilepsy. ${ }^{65,66}$

Huang et $\mathrm{al}^{67}$ developed a chip to release AED ethosuximide (ESM). The chip was fabricated upon depositing the drug-carrying core-shell magnetic NPs into an electrically conductive flexible polyethylene terephthalate (PET) substrate using electrophoretic method. The application of magnetic field triggers the release of the drug from the chip by various release profiles. In vivo studies revealed the reduction in spike wave discharge upon the release of drug and thus suggested the use of chip for reducing the seizures in epileptic patients. The flexible chip is more effective when compared with the conventional drug delivery system due to the improvement in dosage, broader versatility in elution pattern and easy surgery. Figure 5 shows the concept of sandwich structure for drug delivery chip.

Polymeric NPs are important in the treatment of epilepsy, due to their higher stability in biological fluids, and the method adopted for the synthesis is also easier. ${ }^{68} \mathrm{NPs}$ ' surface charge plays an important role in their distribution in brain, and the charge can be altered during the process of fabrication. The drugs that are either hydrophobic or hydrophilic can be incorporated into the NPs during the synthesis process. After the drugs are loaded, they are transported by desorption and diffusion, or through polymer degradation/erosion, NPs are employed as the drug delivery system during the nasal route of drug administration. This is mainly because of their efficiency to travel across the BBB, and their increased transportation rate reduces the dosage frequency. ${ }^{69,70}$ Surfacecharged NP system coated by dipalmitoylphosphatidyl choline and cholesterol was analyzed with in vitro model which contains rat astrocytes and bovine brain capillary endothelial cells.

The NPs showed enhanced efficiency in crossing the barrier when compared with control NPs. An NP system of

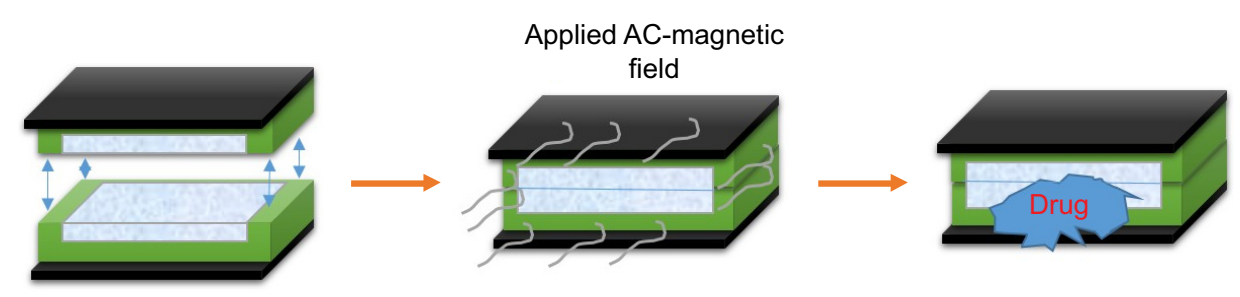

Figure $\mathbf{5}$ The concept of sandwich structure for drug delivery chip. Abbreviation: AC, alternating current. 
surface coated by poly(butylcyanoacrylate) with polysorbate 80 was developed as a noncompetitive $N$-methyl-D-aspartate (NMDA) receptor antagonist, and MRZ 2/576 (8-chloro4-hydroxy-1-oxo-1,2-dihydropyridazino[4,5-b]quinoline5-oxide choline salt) was incorporated into the NMDA. When the NP comes in contact with blood, the polysorbate 80 coating on the NPs gets attached to the apolipoproteins E and $\mathrm{B} .{ }^{71}$ The newly developed lipoprotein coating composed of natural lipoproteins crosses the BBB through low-density lipoprotein receptors present on the brain capillary endothelial cells. Although the drug delivery system is capable of traveling across BBB, the major limitations of this method are as follows:

- NP degradation rate is higher.

- The therapeutic efficiency of the drug is decreased.

- Polysorbate 80 coating desorption from the NPs occurs.

- Toxicity resulted due to the polymer or from the surfactant used.

Hsiao et $\mathrm{al}^{73}$ prepared a potential drug delivery system by preparing a thermo-gelling injectable nanogel. The therapeutic efficiency of nanogels loaded with ESM was found to suppress the spike-wave discharges in Long Evans rat model.
The gel clearance at the site of administration was observed noninvasively using MRI.

Thus, it was found that the nanogels are shown to be used as excellent injectable depot drug delivery system. ${ }^{73}$ Development of an effective drug delivery system is essential for the release of AEDs to suppress the neuronal discharge in epilepsy patients. Ying et $\mathrm{al}^{72}$ developed electro-responsive hydrogel NPs (ERHNPs) that were designed and modified with the angiopep-2 (ANG) peptide, a brain-targeting ligand, which is a low-density lipoprotein receptor-related protein (Figure 6) and facilitates the release of phenytoin (PHT) and AED. The drug release from the ERHNPs is triggered by the application of external electric field. The amygdale kindling model was employed for the determination of its antiepileptic effect. From the results (Figure 7), it was found that ANGERHNPs were capable to release the drug.

$\mathrm{Fu}$ et $\mathrm{al}^{74}$ worked on theranostic application of NPs in acute temporal lobe epilepsy (TLE) with functionalized superparamagnetic iron oxide (SPION). Anti-IL-1 $\beta$ monoclonal antibody (mAb) was attached to functionalized SPION and was used for MRI diagnosis and targeted therapy. In MRI imaging, the particles were found to be concentrated in the

\section{A}

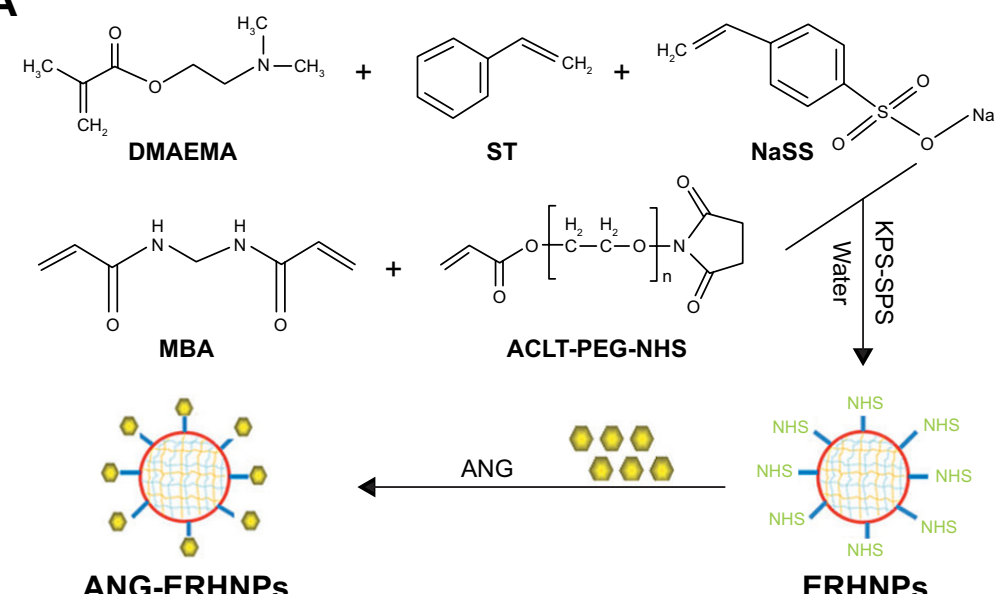

B

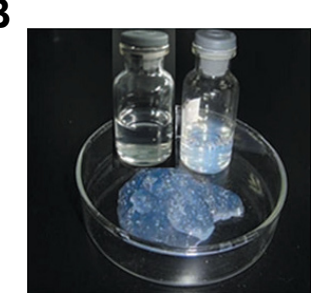

C

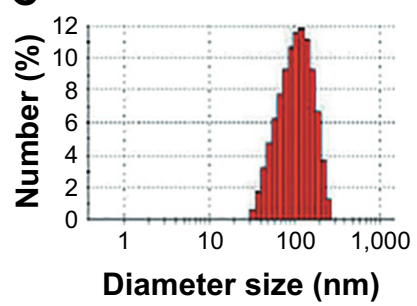

D

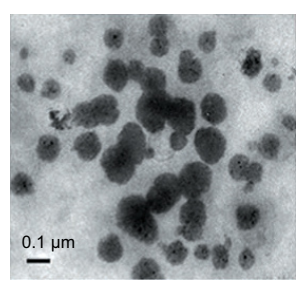

Figure 6 Synthesis and characterization of the ANG-ERHNPs.

Notes: (A) Synthesis of the ANG-ERHNPs. (B) Photograph of the ERHNP dispersion. (C) Particle size distribution of the ERHNPs. (D) TEM image of the ERHNPs. Adapted from Ying X, Wang Y, Liang J, et al. Angiopep-conjugated electro-responsive hydrogel nanoparticles: therapeutic potential for epilepsy. Angew Chem Int Ed Engl. 20I4;53(46): I2436-I2440. Copyright (20I4) with permission from John Wiley and Sons. ${ }^{72}$

Abbreviations: ACLT-PEG-NHS, acrylate-poly(ethylene glycol)-Nhydroxysuccinimidylester; ANG, angiopep-2; DMEMEA, 2-dimethylamino ethyl methacrylate; ERHNPs, electro-responsive hydrogel NPs; KPS-SPS, different sulfonates; MBA, N,N'-methylene bisacrylamide; NPs, nanoparticles; ST, Styrene; TEM, transmission electron microscopy. 
A

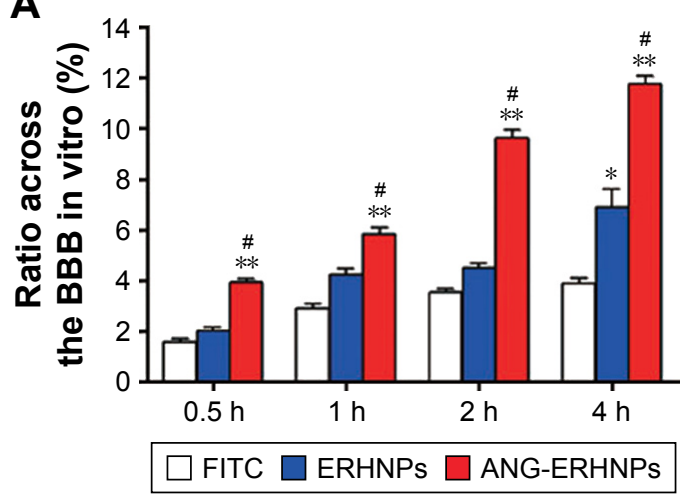

B

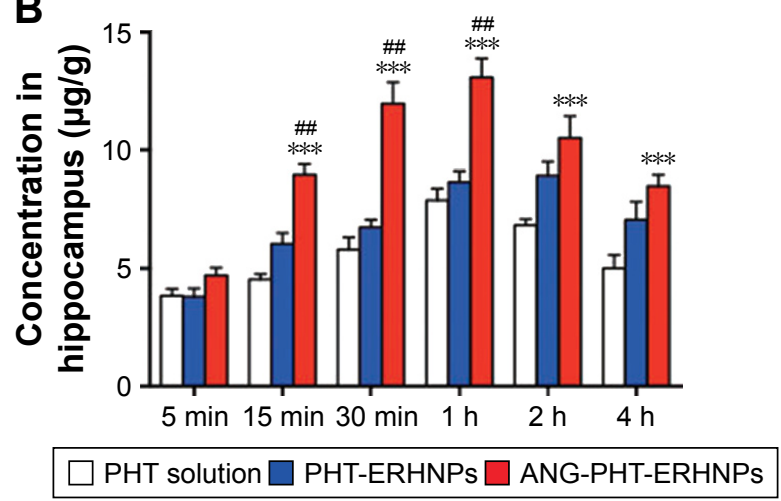

C
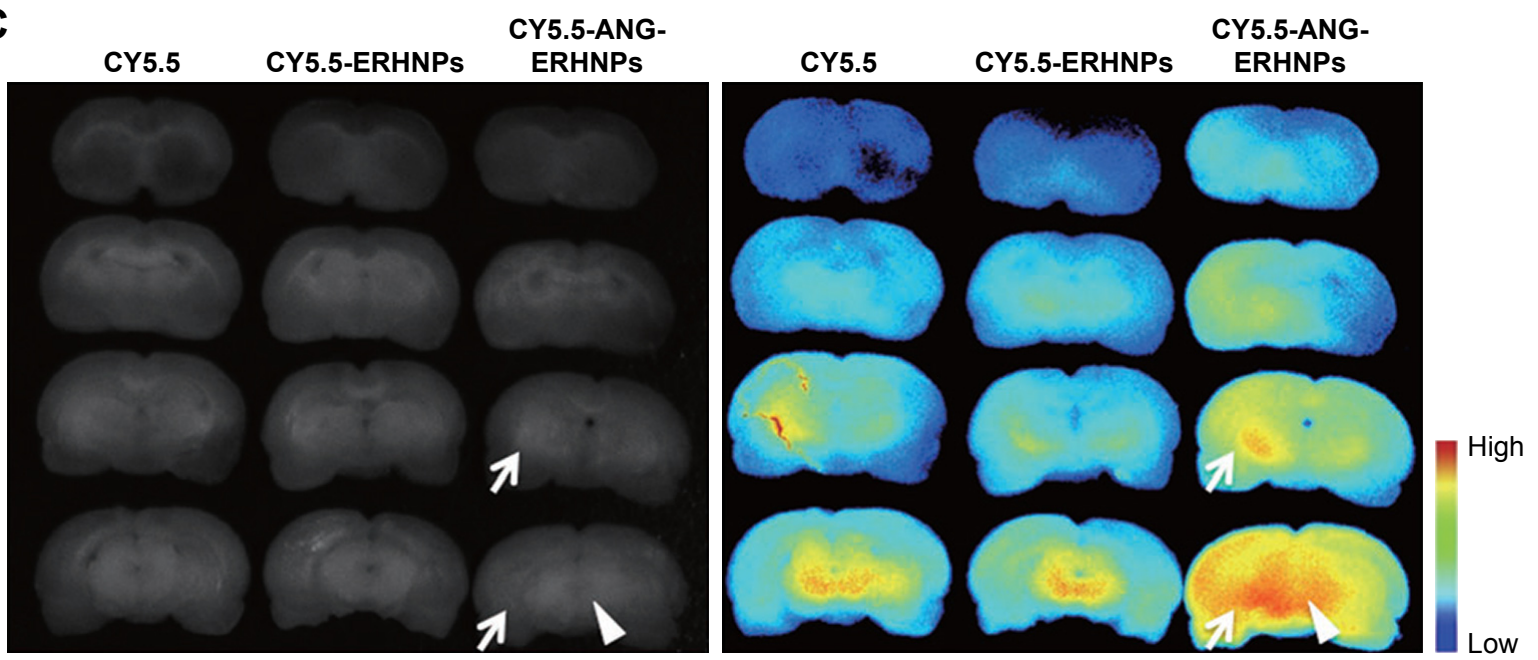

Figure 7 (A) The transport percentage of FITC, ERHNPs and ANG-ERHNPs across an in vitro BBB model at various times. $* p<0.05$, $* * p<0.01$ compared with FITC, ${ }^{\#} p<0.05$ represents the difference between the ERHNPs and ANG-ERHNPs ****\#Anova results. (B) PHT concentrations in the hippocampus after the administration of PHT, PHT-ERHNPs and ANG-PHT-ERHNPs at the dose of $50 \mathrm{mg} \cdot \mathrm{kg}^{-1}$ for various times. (C) Ex vivo fluorescence imaging of coronal brain slices. Arrows indicate the hippocampus, and triangles indicate the brainstem. Adapted from Ying X, Wang Y, Liang J, et al. Angiopep-conjugated electro-responsive hydrogel nanoparticles: therapeutic potential for epilepsy. Angew Chem Int Ed Engl. 20I4;53(46):I2436-12440. Copyright (2014) with permission from John Wiley and Sons. ${ }^{72}$

Abbreviations: ANG, angiopep-2; BBB, blood-brain barrier; ERHNPs, electro-responsive hydrogel NPs; FITC, fluorescein isothiocyanate; NPs, nanoparticles; $\mathrm{PHT}$, phenytoin.

astrocytes and neurons in epileptogenic tissues, simultaneously providing therapeutic action.

The researchers focused on the development of AED delivery systems, successful systems regarding the development of chip upon depositing the drug-carrying core-shell magnetic NPs. Polymeric NPs are found to be important in the treatment of epilepsy.

\section{PD}

It is an ND caused due to the loss of dopamine (DA)-producing neurons that project from the substantia nigra pars compacta to the corpus striatum. The loss of DA results in bradykinesia, rigidity and resting tremor, as DA is involved in the transmission of electric signals to the normal physical motion. Levodopa is also an efficient drug commonly used in the treatment of PD. However, it may cause a long-term side effect. This ultimately leads to pulsatile production of DA receptors. ${ }^{75}$

The formation and accumulation of Lewy bodies (cytoplasmic eosinophilic inclusions) made of a protein termed as alpha synuclein are observed in most neuronal populations in PD patients. These Lewy bodies are first formed at the brain stem and olfactory bulb which later spread to cortex and nigra. This ultimately leads to the dysfunctioning of neurotransmitter systems such as adrenergic, cholinergic and serotonergic.

Some of the administration routes employed during the delivery of drugs are as follows:

- The administration of drugs via mouth takes a long time to reach the DA receptors. Some of the important parameters such as gastric $\mathrm{pH}$, dietary proteins and constipation influence the bioavailability of drugs. 
- Other routes of administration are rectal, pulmonary and sublingual, and they are not fully effective.

- Infusion pumps are used to deliver apomorphine through subcutaneous routes; also through skin patches, the drugs are available.

- In rotigotine patch delivery method, the drug is contained in elastic vessels released into silicon matrix and by the ionic gradients; they are transported and evenly absorbed in the epidermis. ${ }^{76-79}$

Even though continuous stimulation of DA receptors is provided by these routes of administration, the targeted and localized drug delivery is still a challenge to the researchers. If the drug is not distributed efficiently, the healthy tissues are affected which results in dyskinesias and psychiatric problems.

Several glial cells such as astrocytes and microglia play an important role in the PD etiology. The most abundant glia in the brain is astrocytes, and they provide support and protection and eliminate toxins produced by neurons. Microglias are the immune cells present in the CNS, and they become active in response to the various factors. The microglia trigger local inflammation process that prevents excessive damage. The microglial cells prevent the spread of infection and elimination of dead cells and debris. ${ }^{42,80}$

From the diagnosis of PD patients' brain, it was noticed that the level of astrocytes present was lower in substantia nigra due to the various downstream processing in brain. ${ }^{81-83}$ NDs are multisystemic in nature and cause difficulties in treating the disorders. The advancement in the field of nanotechnology helps in overcoming the obstacles placed in the treatment of ND. The ability of NPs to cross the BBB in the in vitro and in vivo studies makes them a suitable choice. Polymeric NPs are employed for the effective treatment of PD.

The main advantages of using these NPs are:

- Alternation in the chemical properties.

- Targeted drug delivery design.

- Increase in bioavailability.

- The drugs loaded are protected from the enzyme degradation.

- The physiochemical properties of the drugs were protected, thus enabling their transportation across the BBB. ${ }^{75}$

Clinically osmotic disruption was adopted to infiltrate the $\mathrm{BBB}$ for short time and allow the entry of various neuroactive molecules to the brain parenchyma. The major limitations of osmotic disruption of BBB are the potential morbidity, causing discomfort to the patient. Recent study articles show that the focus is mainly on nanotechnology for the development of nonviral vector system for effective delivery of therapeutic genes into the cells. ${ }^{84,85}$
Organically modified amino-functionalized silica NPs were used as a nonviral vector; they bind and protect the plasmid DNA against enzymatic degradation. This initiates the effective transport of genetic material to the nucleus of the cell. ${ }^{86}$

Yurek et $\mathrm{al}^{87}$ reported on the development of nonviral technique for the transfer of gene encoding GDNF to striatal cells. They suggested from the data analysis of morphological, protein, behavioral measures that compacted $p G D N F$ $D N P s$ which were injected into the striatum, resulted in the overexpressions of GDNF protein in the transfected cells at the appropriate levels for them to provide neurotrophic support to the grafted embryonic DA neurons.

Wen et $\mathrm{al}^{88}$ reported on the improvement in brain drug delivery system through nose and noticed a reduction in immunogenicity by the drug delivery system modified with traditional lectin. Upon double emulsion method, odorranalectin (OL)-modified NPs were obtained by mixing the synthesized OL-conjugated PEG-PLGA) (OL-PEG-PLGA) to the unmodified PEG-PLGA. Hemagglutination test was performed for confirming the biorecognitive activity of OL on the surface of NPs. The result as shown in Figure 8 indicates that the conjugated OL retains hemagglutinating activity, and the process of OL-NP synthesis has influence on bioactivity. In vivo fluorescence imaging technique by using 1,1'-Dioctadecyl-3,3,3',3'-tetramethylindotricarbocyanine iodide as a tracer was employed to investigate the nose-brain delivery characteristics of OL-conjugated NPs.

In addition, NPs were incorporated with a macromolecular model drug urocortin peptide, and its therapeutic efficiency toward hemiparkinsonian rats was evaluated along with the intranasal administration by rotation behavior test, tyrosine hydrolase and neurotransmitter determination test. As a result, it was concluded that the brain delivery of NPs increased the OL modification and also thus enhanced the therapeutic efficiency of urocortin-loaded NPs in PD.

A similar study was reported by $\mathrm{Hu}$ et $\mathrm{al}^{89}$ on a novel biodegradable drug delivery system method by thiolating and conjugating lactoferrin to distal maleimide function surrounding a pegylated NP to form lactoferrin (LF)-NPs. By performing the transmission electron microscopy (TEM) and enzyme-linked immunosorbent assay (ELISA) analysis, the presence of LF on the LF-NP surface was confirmed. Coumarin- 6 was utilized as a fluorescent probe for the quantitative and qualitative uptake studies; more pronounced accumulation of LF-NP in bEnd. 3 cells was found when compared with the unconjugated NPs. Increase in the uptake of LF-NP occurred through additional clathrin-mediated endocytosis; it was identified by performing uptake inhibition studies. 
A

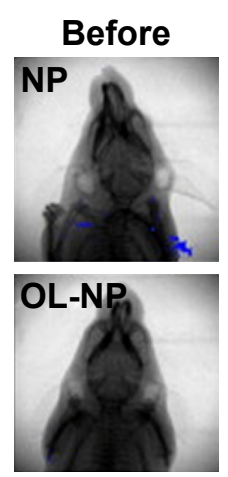

$2 \mathrm{~h}$
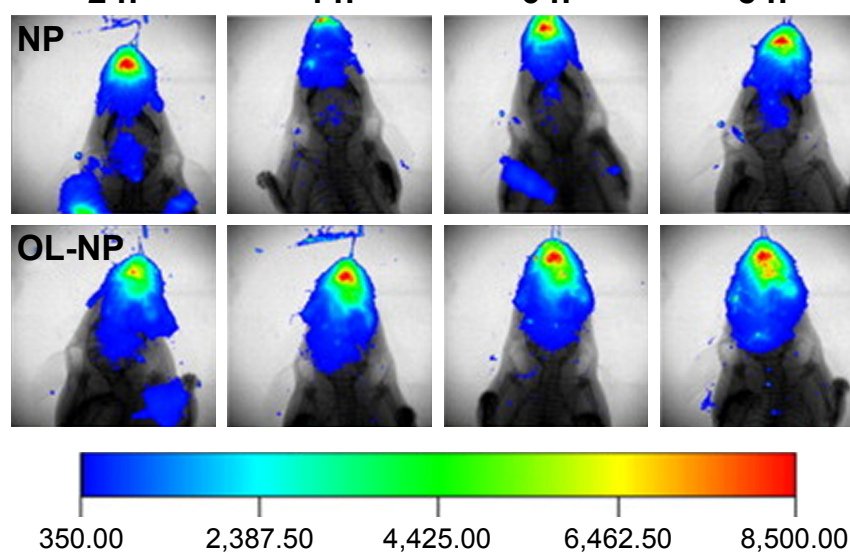

B

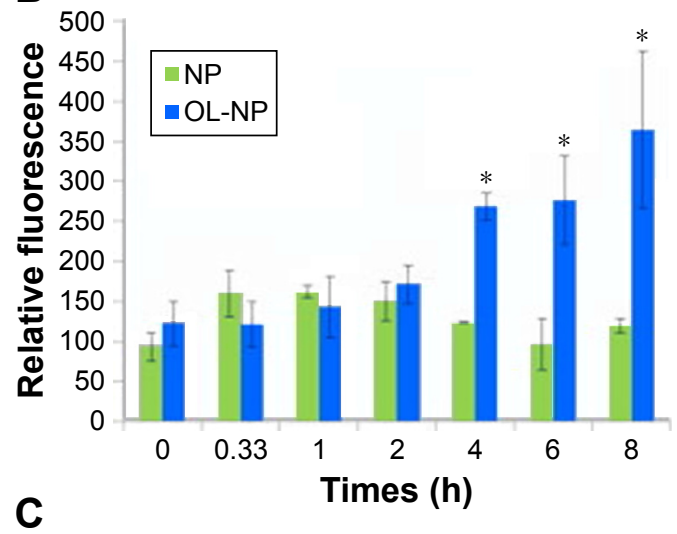

C

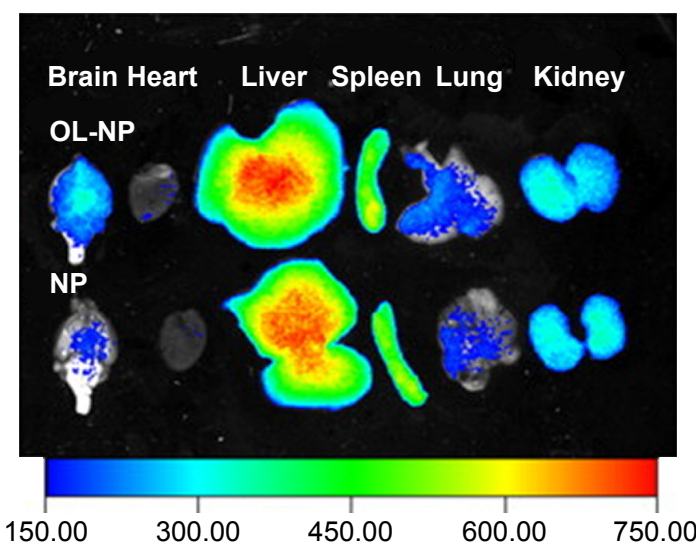

Figure 8 The brain delivery study of OL-NP by in vivo imaging system.

Notes: (A) Fluorescence images of mouse upper half-body overlaid on the X-ray images following intranasal administration of DiR-loaded NPs and OL-NPs at different time points. (B) Semiquantitative results of the fluorescence intensity in the brain region. $* p<0.05$, significantly different. (C) Fluorescence images of major organs overlaid on the white light images at $8 \mathrm{~h}$ after intranasal administration of DiR-loaded NPs and OL-NPs to mice. Adapted from J Control Release. Wen Z, Yan Z, Hu K, et al. Odorranalectinconjugated nanoparticles: preparation, brain delivery and pharmacodynamic study on Parkinson's disease following intranasal administration. 20I I; I5I (2): I II-I38. Copyright (C) 201 I Elsevier B.V. All rights reserved, with permission from Elsevier. ${ }^{88}$

Abbreviations: DiR, I, I'-Dioctadecyl-3,3,3',3'-tetramethylindotricarbocyanine iodide; NPs, nanoparticles; OL, odorranalectin.

In conclusion, the modified traditional lectin was found to be a promising approach. In addition, the brain delivery of NPs increased the OL modification and also thus enhanced the therapeutic efficiency of urocortin-loaded NPs in PD. In addition, a novel biodegradable drug delivery system method by thiolating and conjugating lactoferrin to distal maleimide function surrounding a pegylated NP to form LF-NPs was found to be a successful method in the treatment of PD.

\section{HD}

HD is a neurodegenerative disease that mainly affects the cortical neurons and striatal (caudate nucleus and putamen) medium spiny neurons (composed by GABAergic neuron). Its pathophysiological features are known by the expression of muHTT protein along with an abnormally long polyglutamine (poly Q) tract ( $>40 \mathrm{Q}$ ) which is close to its $\mathrm{N}$ terminus. muHTT can sequester various proteins which leads to cellular dysfunction involving energy deregulation, transcription deregulation and myotic synaptic dysfunction. The disease is characterized by involuntary choreiform (rapid movements, anxiety and progressive motor and cognitive movement), mood alterations, oxidative injury and transcriptional dysregulation, usually leading to death. From the observations of the biomaterials for neurodegenerative disease patients, the existence of mitochondrial defects and oxidative stress was detected. Moreover, the dysfunctions in mitochondria can also lead to neuronal loss and lower the activity of electron transport chain in HD. It is also accompanied by the neurochemical alterations in receptors such as DA and adenosine and also in glutamate receptors. Since metabolic abnormalities are common features, HD patients are prone to a higher risk of diabetes development. The alterations in intracellular signaling pathway which are responsible for controlling neuronal survival are also associated with the development of HD. ${ }^{90-95}$

The exact causative pathway responsible for HD remains unknown. But mitochondrial dysfunction was identified to be involved in HD pathogenesis. A method should be developed to overcome mitochondrial impairments for successful therapy. Sandhir et al developed a solid 
lipid NP encapsulated with curcumin (C-SLN) to ameliorate 3-nitropropionic acid induced HD in rats. From the results of succinate dehydrogenase (SDH) staining and 3-(4,5-dimethylthiazolyl-2)-2,5-dihenyltetrazolium bromide (MTT) assay of striatum, the complex II activity was found to be decreased. To measure the changes in Nrf2 expression in 3-nitropropionic acid-treated rats, quantitative polymerase chain reaction (PCR) was performed. Increase in Nrf2 mRNA levels (3.309 \pm 0.046$)$ was found in the treatment with 3-nitropropionic acid when compared with the control $(0.924 \pm 0.053)$. Moreover, in the striatal tissue of C-SLN administered, rat's upregulated mRNA level expression $(1.621 \pm 0.049)$ was noticed. From the Western blot analysis, it was found that upon comparison with C-SLN-administered animals, less expression of Nrf2 was identified in nuclear fraction of 3-nitropropionic acid-treated animals. However, on the other side, on comparison with 3-nitropropionic acid-treated animals to C-SLN-treated animals showed increase in the cytosolic and nuclear fractions. ${ }^{96}$

Bhatt et $\mathrm{al}^{97}$ studied the use of solid lipid NPs (SLNs) as effective system for drug delivery to enhance the brain-targeting efficiency of rosmarinic acid (RA) through intranasal administration. The mean size of the RA-loaded SLN was found to be $149.2 \pm 3.2 \mathrm{~nm}$, zeta potential was $-38.27 \mathrm{mV}$ and its entrapment efficiency was observed as $61.9 \% \pm 2.2 \%$. Based on the organ distribution studies, the brain drug concentration was found to be $5.69 \mu \mathrm{g}$. On comparison with the IV route, the nasal route of drug administration has prevented non-required transport and metabolism in other body parts. From the results, it was confirmed that the RA-loaded SLNs can emerge as a promising tool for the effective management of HD.

In conclusion, the use of SLNs is an effective system for drug delivery to enhance the brain-targeting efficiency of RA through intranasal administration. C-SLN was also found to be a successful carrier system in the treatment of HD.

\section{Conclusion}

The application of NPs in the therapeutics and diagnostics appears to be promising methodology in recent years. Multifunctional nano-carriers employed for the targeted drug delivery, in vivo imaging, bring many essential treatments for neurodegenerative diseases. The scientific paradigm for the adverse activity of NPs is lacking, and only few ideas were obtained regarding the interaction of NPs with cells, organs and organism. It is very important to understand the bio-responses to the nanomaterials. To achieve success in the nanomedicine, the most important factors such as targeting, imaging and functionalization should be implemented. Various new methods have been developed for the transport of drug to the CNS. But the transport of the drug material has been limited by the presence of BBB. The development in the field of nanotechnology has promising application toward the treatment of CNS-related disorders such as AD, PD and epilepsy. The advancements in the field of nanotechnology have created a revolution in the successful transfer of drug across the BBB. The drugs are either encapsulated or attached to the surface of the nano-carriers through certain modification process. As such, NPs are a boon to the field of nanotechnology, and they differ from other bulk materials due to their size-controlled physical, chemical, biological and optical properties. The contribution of these NPs in the transport of drugs across the BBB in the treatment of NDs is unparalleled. Yet, additional studies must be carried out to overcome the safety and toxicity issues against the use of NPs. More studies are needed regarding HD, ie, in vivo studies are required for significant tracking of causes of the disease and improving the drug-delivering strategy.

\section{Acknowledgments}

PP and BG acknowledge the support from Lee Kong Chian School of Medicine, Nanyang Technological University Start-up Grant. GA acknowledges the support from University Grants Commission - Special Assistance Program, New Delhi, for the facility available in the Department of Animal Science, Bharathidasan University, Tiruchirappalli, India.

\section{Disclosure}

The authors report no conflicts of interest in this work.

\section{References}

1. The Royal Society \& The Royal Academy of Engineering. Nanomanufacturing and the industrial application of nanotechnologies; 2004. Available from: http://www.nanotec.org.uk/report/chapter4.pdf. Accessed June 12, 2018.

2. Neuwelt EA, Bauer B, Fahlke C, et al. Engaging neuroscience to advance translational research in brain barrier biology. Nat Rev Neurosci. 2011;12(3):169-182

3. Weksler BB, Subileau EA, Perrière N, et al. Blood-brain barrier-specific properties of a human adult brain endothelial cell line. FASEB J 2005;19(13):1872-1874.

4. Davson H, Segal MB. Physiology of the CSF and Blood-Brain Barriers. Boca Raton: CRC Press; 1996:11.

5. Bolognesi ML. From imaging agents to theranostic drugs in Alzheimer's disease. Reference Module in Chemistry, Molecular Sciences and Chemical Engineering. New York: Elsevier; 2017:74-106.

6. Sikkandhar MG, Nedumaran AM, Ravichandar R, etal. Theranostic probes for targeting tumor microenvironment: an overview. Int $J$ Mol Sci. 2017;18(5):E1036.

7. Sridhar S, Mishra S, Gulyás M, Padmanabhan P, Gulyás B. An overview of multimodal neuroimaging using nanoprobes. Int J Mol Sci. 2017; $18(2): 311$

8. Moreno-Gonzalez I, Soto C. Misfolded protein aggregates: Mechanisms, structures and potential for disease transmission. Semin Cell Dev Biol. 2011;22(5):482-487.

9. Sweeney P, Park H, Baumann M, et al. Protein misfolding in neurodegenerative diseases: implications and strategies. Transl Neurodegener 2017;6(1):6. 
10. Begley DJ, Kreuter J. Do ultra-low frequency (ULF) magnetic fields affect the blood-brain barrier? In: Holick MF, Jung EG, editors. Biologic Effects of Light 1998. Boston, MA: Springer US; 1999:297-301.

11. Juillerat-Jeanneret L. The targeted delivery of cancer drugs across the blood-brain barrier: chemical modifications of drugs or drugnanoparticles? Drug Discov Today. 2008;13(23-24):1099-1106.

12. Begley DJ, Brightman MW. Structural and functional aspects of the blood-brain barrier. Prog Drug Res. 2003;61:39-78.

13. Olivier J-C. Drug transport to brain with targeted nanoparticles. NeuroRx. 2005;2(1):108-119.

14. Gabathuler R. Approaches to transport therapeutic drugs across the blood-brain barrier to treat brain diseases. Neurobiol Dis. 2010;37(1): $48-57$.

15. Hans ML, Lowman AM. Biodegradable nanoparticles for drug delivery and targeting. Curr Opin Solid State Mater Sci. 2002;6(4): 319-327.

16. Persidsky Y, Ramirez SH, Haorah J, Kanmogne GD. Blood-brain barrier: structural components and function under physiologic and pathologic conditions. J Neuroimmune Pharmacol. 2006;1(3):223-236.

17. Singh R, Lillard JW. Nanoparticle-based targeted drug delivery. Exp Mol Pathol. 2009;86(3):215-223.

18. Choi HS, Liu W, Misra P, et al. Renal clearance of nanoparticles. Nat Biotechnol. 2007;25(10):1165.

19. Bhadra D, Bhadra S, Jain P, Jain N. Pegnology: a review of PEG-ylated systems. Pharmazie. 2002;57(1):5-29.

20. Wilson B, Samanta MK, Santhi K, Kumar KPS, Paramakrishnan N, Suresh B. Poly (n-butylcyanoacrylate) nanoparticles coated with polysorbate 80 for the targeted delivery of rivastigmine into the brain to treat Alzheimer's disease. Brain Res. 2008;1200:159-168.

21. Chung H, Choi H, Kim D, Jeong S, Kim J. Size dependence of excitation-energy-related surface trapping dynamics in $\mathrm{PbS}$ quantum dots. J Phys Chem C. 2015;119(13):7517-7524.

22. Borm PJ, Robbins D, Haubold S, et al. The potential risks of nanomaterials: a review carried out for ECETOC. Part Fibre Toxicol. 2006;3:11

23. Roduner E. Size matters: why nanomaterials are different. Chem Soc Rev. 2006;35(7):583-592.

24. Das GK, Zhang Y, D'Silva L, et al. Single-phase Dy2O3:Tb3+ nanocrystals as dual-modal contrast agent for high field magnetic resonance and optical imaging. Chem Mater. 2011;23(9):2439-2446.

25. Janib SM, Moses AS, MacKay JA. Imaging and drug delivery using theranostic nanoparticles. Adv Drug Deliv Rev. 2010;62(11):1052-1063.

26. Chen Z. Small-molecule delivery by nanoparticles for anticancer therapy. Trends Mol Med. 2010;16(12):594-602.

27. Li W, Luo R, Lin X, et al. Remote modulation of neural activities via near-infrared triggered release of biomolecules. Biomaterials. 2015;65: 76-85.

28. Müller RH, Jacobs C, Kayser O. Nanosuspensions as particulate drug formulations in therapy: rationale for development and what we can expect for the future. Adv Drug Deliv Rev. 2001;47(1):3-19.

29. Wilson B, Samanta MK, Santhi K, Kumar KPS, Paramakrishnan N, Suresh B. Targeted delivery of tacrine into the brain with polysorbate 80-coated poly(n-butylcyanoacrylate) nanoparticles. Eur J Pharm Biopharm. 2008;70(1):75-84.

30. Kulkarni PV, Roney CA, Antich PP, Bonte FJ, Raghu AV. Quinolinen-butylcyanoacrylate-based nanoparticles for brain targeting for the diagnosis of Alzheimer's disease. Wiley Interdiscip Rev Nanomed Nanobiotechnol. 2010;2(1):35-47.

31. Wilson B, Samanta MK, Santhi K, Kumar KPS, Ramasamy M, Suresh B. Chitosan nanoparticles as a new delivery system for the anti-Alzheimer drug tacrine. Nanomedicine. 2010;6(1):744-750.

32. Zhao X, Li F, Li Y, et al. Co-delivery of HIF $1 \alpha$ siRNA and gemcitabine via biocompatible lipid-polymer hybrid nanoparticles for effective treatment of pancreatic cancer. Biomaterials. 2015;46:13-25.

33. Osminkina LA, Nikolaev AL, Sviridov AP, et al. Porous silicon nanoparticles as efficient sensitizers for sonodynamic therapy of cancer. Microporous Mesoporous Mater. 2015;210:169-175.
34. Zhao L, Zhou Y, Gao Y, et al. Bovine serum albumin nanoparticles for delivery of tacrolimus to reduce its kidney uptake and functional nephrotoxicity. Int J Pharm. 2015;483(1-2):180-187.

35. Haller E, Lindner W, Lämmerhofer M. Gold nanoparticle-antibody conjugates for specific extraction and subsequent analysis by liquid chromatography-tandem mass spectrometry of malondialdehydemodified low density lipoprotein as biomarker for cardiovascular risk. Anal Chim Acta. 2015;857:53-63.

36. Fu YC, Fu TF, Wang HJ, et al. Aspartic acid-based modified PLGAPEG nanoparticles for bone targeting: in vitro and in vivo evaluation. Acta Biomater. 2014;10(11):4583-4596.

37. Kumar GS, Kulkarni A, Khurana A, Kaur J, Tikoo K. Selenium nanoparticles involve HSP-70 and SIRT1 in preventing the progression of type 1 diabetic nephropathy. Chem Biol Interact. 2014;223: $125-133$.

38. Miguel L, Noiray M, Surpateanu G, Iorga BI, Ponchel G. De \& Poly ( $\gamma$-benzyl-1-glutamate)-PEG-alendronate multivalent nanoparticles for bone targeting. Int J Pharm. 2014;460(1-2):73-82.

39. Yilmaz A, Rösch S, Klingel K, et al. Magnetic resonance imaging (MRI) of inflamed myocardium using iron oxide nanoparticles in patients with acute myocardial infarction - preliminary results. Int J Cardiol. 2013; 163(2):175-182.

40. Badr G, Al-Sadoon MK, Rabah DM. Therapeutic efficacy and molecular mechanisms of snake (Walterinnesia aegyptia) venom-loaded silica nanoparticles in the treatment of breast cancer- and prostate cancerbearing experimental mouse models. Free Radic Biol Med. 2013;65: $175-189$.

41. Gao Y, Gu W, Chen L, Xu Z, Li Y. The role of daidzein-loaded sterically stabilized solid lipid nanoparticles in therapy for cardio-cerebrovascular diseases. Biomaterials. 2008;29(30):4129-4136.

42. Kaur IP, Bhandari R, Bhandari S, Kakkar V. Potential of solid lipid nanoparticles in brain targeting. $J$ Control Release. 2008;127(2):97-109.

43. Faraji AH, Wipf P. Nanoparticles in cellular drug delivery. Bioorg Med Chem. 2009;17(8):2950-2962.

44. Kreuter J, Ramge P, Petrov V, et al. Direct evidence that polysorbate80-coated poly(butylcyanoacrylate) nanoparticles deliver drugs to the CNS via specific mechanisms requiring prior binding of drug to the nanoparticles. Pharm Res. 2003;20(3):409-416.

45. Volicer L. Management of severe Alzheimer's disease and end-of-life issues. Clin Geriatr Med. 2001;17(2):377-391.

46. Forchetti CM. Treating Patients With Moderate to Severe Alzheimer's Disease: Implications of Recent Pharmacologic Studies. Prim Care Companion J Clin Psychiatry. 2005;7(4):155-161.

47. Selkoe DJ, Schenk D. Alzheimer's disease: molecular understanding predicts amyloid-based therapeutics. Annu Rev Pharmacol Toxicol. 2003;43(1):545-584.

48. Mahmoudi M, Shokrgozar MA. Multifunctional stable fluorescent magnetic nanoparticles. Chem Commun. 2012;48(33):3957-3959.

49. Helzner EP, Scarmeas N, Cosentino S, Tang MX, Schupf N, Stern Y. Survival in Alzheimer disease: a multiethnic, population-based study of incident cases. Neurology. 2008;71(19):1489-1495.

50. Xie J, Brayne C, Matthews FE, Medical Research Council Cognitive Function and Ageing Study Collaborators. Survival times in people with dementia: analysis from population based cohort study with 14 year follow-up. BMJ. 2008;336(7638):258-262.

51. Allinson TM, Parkin ET, Turner AJ, Hooper NM. ADAMs family members as amyloid precursor protein alpha-secretases. J Neurosci Res. 2003;74(3):342-352.

52. Dickson DW, Crystal HA, Bevona C, Honer W, Vincent I, Davies P. Correlations of synaptic and pathological markers with cognition of the elderly. Neurobiol Aging. 1995;16(3):285-298.

53. Terry RD, Masliah E, Salmon DP, et al. Physical basis of cognitive alterations in Alzheimer's disease: synapse loss is the major correlate of cognitive impairment. Ann Neurol. 1991;30(4):572-580.

54. Begley DJ. Delivery of therapeutic agents to the central nervous system: the problems and the possibilities. Pharmacol Ther. 2004; 104(1):29-45. 
55. Roney C, Kulkarni P, Arora V, et al. Targeted nanoparticles for drug delivery through the blood-brain barrier for Alzheimer's disease. J Control Release. 2005;108(2):193-214.

56. Tanifum EA, Dasgupta I, Srivastava M, et al. Intravenous delivery of targeted liposomes to amyloid- $\beta$ pathology in APP/PSEN1 transgenic mice. PLoS One. 2012;7(10):e48515.

57. Cui Z, Lockman PR, Atwood CS, et al. Novel D-penicillamine carrying nanoparticles for metal chelation therapy in Alzheimer's and other CNS diseases. Eur J Pharm Biopharm. 2005;59(2):263-272.

58. Agyare EK, Curran GL, Ramakrishnan M, Yu CC, Poduslo JF, Kandimalla KK. Development of a smart nano-vehicle to target cerebrovascular amyloid deposits and brain parenchymal plaques observed in Alzheimer's disease and cerebral amyloid angiopathy. Pharm Res. 2008;25(11):2674-2684.

59. Bastus NG, Kogan MJ, Amigo R, et al. Gold nanoparticles for selective and remote heating of $\beta$-amyloid protein aggregates. Mater Sci Eng C. 2007;27(5-8):1236-1240.

60. Zhang C, Chen J, Feng C, et al. Intranasal nanoparticles of basic fibroblast growth factor for brain delivery to treat Alzheimer's disease. Int J Pharm. 2014;461(1-2):192-202.

61. Zhang C, Wan X, Zheng X, et al. Dual-functional nanoparticles targeting amyloid plaques in the brains of Alzheimer's disease mice. Biomaterials. 2014;35(1):456-465.

62. Li Y, Xu D, Ho SL, Li HW, Yang R, Wong MS. A theranostic agent for in vivo near-infrared imaging of $\beta$-amyloid species and inhibition of $\beta$-amyloid aggregation. Biomaterials. 2016;94:84-92.

63. Fisher RS, Ho J. Potential new methods for antiepileptic drug delivery. CNS Drugs. 2002;16(9):579-593.

64. De Tiège X, Laufs H, Boyd SG, et al. EEG-fMRI in children with pharmacoresistant focal epilepsy. Epilepsia. 2007;48(2):385-389.

65. Moody WJ, Futamachi KJ, Prince DA. Extracellular potassium activity during epileptogenesis. Exp Neurol. 1974;42(2):248-263.

66. Rutecki PA, Lebeda FJ, Johnston D. Epileptiform activity induced by changes in extracellular potassium in hippocampus. J Neurophysiol. 1985;54(5):1363-1374.

67. Huang WC, Hu SH, Liu KH, Chen SY, Liu DM. A flexible drug delivery chip for the magnetically-controlled release of anti-epileptic drugs. J Control Release. 2009;139(3):221-228.

68. Couvreur P, Vauthier C. Nanotechnology: intelligent design to treat complex disease. Pharm Res. 2006;23(7):1417-1450.

69. Birnbaum DT, Kosmala JD, Brannon-Peppas L. Optimization of preparation techniques for poly(lactic acid-co-glycolic acid) nanoparticles. J Nanoparticle Res. 2000;2(2):173-181.

70. Ito F, Fujimori H, Makino K. Incorporation of water-soluble drugs in PLGA microspheres. Colloids Surf B Biointerfaces. 2007;54(2): 173-178.

71. Friese A, Seiller E, Quack G, Lorenz B, Kreuter J. Increase of the duration of the anticonvulsive activity of a novel NMDA receptor antagonist using poly (butylcyanoacrylate) nanoparticles as a parenteral controlled release system. Eur J Pharm Biopharm. 2000;49(2):103-109.

72. Ying X, Wang Y, Liang J, et al. Angiopep-conjugated electro-responsive hydrogel nanoparticles: therapeutic potential for epilepsy. Angew Chem Int Ed Engl. 2014;53(46):12436-12440.

73. Hsiao MH, Larsson M, Larsson A, et al. Design and characterization of a novel amphiphilic chitosan nanocapsule-based thermo-gelling biogel with sustained in vivo release of the hydrophilic anti-epilepsy drug ethosuximide. J Control Release. 2012;161(3):942-948.

74. Fu T, Kong Q, Sheng H, Gao L. Value of functionalized superparamagnetic iron oxide nanoparticles in the diagnosis and treatment of acute temporal lobe epilepsy on MRI. Neural Plast. 2016;2016: 2412958.

75. Singh N, Pillay V, Choonara YE. Advances in the treatment of Parkinson's disease. Prog Neurobiol. 2007;81(1):29-44.

76. Johnston TH, Fox SH, Brotchie JM. Advances in the delivery of treatments for Parkinson's disease. Expert Opin Drug Deliv. 2005;2(6):1059-1073.
77. Katzenschlager R, Hughes A, Evans A, et al. Continuous subcutaneous apomorphine therapy improves dyskinesias in Parkinson's disease: a prospective study using single-dose challenges. Mov Disord. 2005; 20(2):151-157.

78. Nyholm D, Nilsson Remahl AI, Dizdar N, et al. Duodenal levodopa infusion monotherapy vs oral polypharmacy in advanced Parkinson disease. Neurology. 2005;64(2):216-223.

79. Bennett JP, Piercey MF. Pramipexole - a new dopamine agonist for the treatment of Parkinson's disease. J Neurol Sci. 1999;163(1):25-31.

80. Patil RR, Yu J, Banerjee SR, et al. Probing in vivo trafficking of polymer/DNA micellar nanoparticles using SPECT/CT imaging. Mol Ther. 2011;19(9):1626-1635.

81. Obeso JA, Rodriguez-Oroz MC, Goetz CG, et al. Missing pieces in the Parkinson's disease puzzle. Nat Med. 2010;16(6):653-661.

82. Mena MA, García de Yébenes J. Glial cells as players in parkinsonism: the "good," the "bad," and the "mysterious" glia. Neuroscientist. 2008; 14(6):544-560.

83. Orr CF, Rowe DB, Halliday GM. An inflammatory review of Parkinson's disease. Prog Neurobiol. 2002;68(5):325-340.

84. Fiandaca MS, Forsayeth JR, Dickinson PJ, Bankiewicz KS. Imageguided convection-enhanced delivery platform in the treatment of neurological diseases. Neurotherapeutics. 2008;5(1):123-127.

85. Neuwelt EA. Mechanisms of disease: the blood-brain barrier. Neurosurgery. 2004;54(1):131-132.

86. Klejbor I, Stachowiak EK, Bharali DJ, et al. ORMOSIL nanoparticles as a non-viral gene delivery vector for modeling polyglutamine induced brain pathology. J Neurosci Methods. 2007;165(2):230-243.

87. Yurek DM, Flectcher AM, Kowalczyk TH, Padegimas L, Cooper MJ. Compacted DNA nanoparticle gene transfer of GDNF to the rat striatum enhances the survival of grafted fetal dopamine neurons. Cell Transplant. 2009;18(10-11):1183-1196.

88. Wen Z, Yan Z, Hu K, et al. Odorranalectin-conjugated nanoparticles: preparation, brain delivery and pharmacodynamic study on Parkinson's disease following intranasal administration. J Control Release. 2011; 151(2):131-138.

89. Hu K, Shi Y, Jiang W, Han J, Huang S, Jiang X. Lactoferrin conjugated PEGPLGA nanoparticles for brain delivery: preparation, characterization and efficacy in Parkinson's disease. Int J Pharm. 2011;415(1-2):273-283.

90. Morrison PJ. Accurate prevalence and uptake of testing for Huntington's disease. Lancet Neurol. 2010;9(12):1147.

91. Pringsheim T, Wiltshire K, Day L, Dykeman J, Steeves T, Jette N. The incidence and prevalence of Huntington's disease: a systematic review and meta-analysis. Mov Disord. 2012;27(9):1083-1091.

92. Wexler NS, Lorimer J, Porter J, et al; U.S.-Venezuela Collaborative Research Project. Venezuelan kindreds reveal that genetic and environmental factors modulate Huntington's disease age of onset. Proc Natl Acad Sci U S A. 2004;101(10):3498-3503.

93. Zuccato C, Cattaneo E. Huntington's Disease. In: Lewin G, Carter B, editors. Neurotrophic Factors. Handbook of Experimental Pharmacology. Volume 220. Berlin, Heidelberg: Springer; 2014.

94. Ross CA, Tabrizi SJ. Huntington's disease: from molecular pathogenesis to clinical treatment. Lancet Neurol. 2011;10(1):83-98.

95. MacDonald ME, Ambrose CM, Duyao MP, et al. A novel gene containing a trinucleotide repeat that is expanded and unstable on Huntington's disease chromosomes. Cell. 1993;72(6):971-983.

96. Sandhir R, Yadav A, Mehrotra A, Sunkaria A, Singh A, Sharma S. Curcumin nanoparticles attenuate neurochemical and neurobehavioral deficits in experimental model of Huntington's disease. Neuromolecular Med. 2014;16(1):106-118.

97. Bhatt R, Singh D, Prakash A, Mishra N. Development, characterization and nasal delivery of rosmarinic acid-loaded solid lipid nanoparticles for the effective management of Huntington's disease. Drug Deliv. 2015;22(7):931-939. 


\section{Publish your work in this journal}

The International Journal of Nanomedicine is an international, peerreviewed journal focusing on the application of nanotechnology in diagnostics, therapeutics, and drug delivery systems throughout the biomedical field. This journal is indexed on PubMed Central,

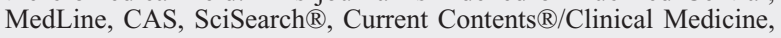

Journal Citation Reports/Science Edition, EMBase, Scopus and the Elsevier Bibliographic databases. The manuscript management system is completely online and includes a very quick and fair peer-review system, which is all easy to use. Visit http://www.dovepress.com/ testimonials.php to read real quotes from published authors.

Submit your manuscript here: http://www.dovepress.com/international-journal-of-nanomedicine-journal 